\title{
Objective Evidence of Pulsus Alternans by Echocardiography in Acute Severe Heart Failure
}

\author{
NILUFAR FATEMA, RAYHAN MASUM MANDAL, JAHANARAARZU, SM AHSAN HABIB, KHURSHED AHMAD, \\ FAZLUR RAHMAN, SAJAL KRISNA BANERJEE, CHAUDHURY MESHKAT AHMED, MD. HARISUL HOQUE
}

Department of Cardiology, BangaBandhu Sheikh Mujib Medical University (BSMMU), Dhaka.

Address of Correspondence: Dr. Nilufar Fatema, Consultant, Department of Cardiology, BangaBandhu Sheikh Mujib Medical University, Dhaka.Email: nilufar.fatema@gmail.com

\begin{abstract}
:
Acute coronary syndrome may leads to heart failure. Severity of heart failure is identified by NYHA classification clinically. Color Doppler echocardiography is the key investigation to identify the acute systolic heart failure. For the quantification of systolic heart failure diagnosis, Ejection fraction (EF) can be measured by Simpson method. EF $>30 \%$ is defined as severe systolic heart failure. Pulsus alternans is found in severe heart failure patients. Pulsus alternans is a poor prognostic sign of severe heart failure patients in acute MI setting. This cross sectional observation study was aimed to identify the objective evidence of Pulsus alternance noninvasively by Echocardiography. Color Doppler echocardiography was done 100 acute coronary syndrome with heart failure NYHA III and IV patients in department of Cardiology, BSMMU, from July 2018 to June 2019. Age 18 to 70 yrs, male is 79 and female is 21. Pulsus alternans was found in 32 patients, 39 had low volume and 29 had normal volume pulse. $50 \%$ patients had ST depression and T inversion and diagnosed as case of Unstable Agina or Non STEMI, 44 had STEMI and 7 had developed new onset of LBBB. 87 Patients who have EF $<30 \%$ was marked as Severe LV systolic dysfunction. Among them, 3 had mild, 14 had moderate and rest 70 had severe Mitral regurgitation. 38 (43.67\%) patients had Doppler alternans in severe LV systolic dysfunction group. P value for Doppler alternans is 0.039 which is significant. Doppler alternans by Echocardiography in Severe LV systolic dysfunction patients showed $49 \%$ sensitivity and $78 \%$ specificity. 38 patients had Severe RV systolic dysfunction by M-mode echo measurement of TAPSE $<10 \mathrm{~mm} .22$ (57.89\%) had Doppler alternans across Tricuspid valve in case of severe $R V$ systolic dysfunction. Sensitivity of Doppler alternans by Doppler Echocardiography in TV severe $R V$ systolic dysfunction is $51 \%$ and specificity for it is $82 \%$.

Early recognition of Doppler alternans can aid in hospitalization, appropriate management and intervention in present treatment, which may change the prognosis.
\end{abstract}

University Heart Journal 2020; 16(2): $92-98$

Introduction:

Acute coronary syndrome often presents with severe chest pain or short of breath. It is a medical emergency that requires prompt diagnosis and care. Treatment goals include improving blood flow, treating complications and preventing future complication according to MAYO clinic. Acute Heart failure occurs when the heart cannot pump enough blood to meet the body's demand. This can be chronic, meaning it happens slowly over time or it can be acute, meaning it happens suddenly. According to a 2014 study, approximately 26 million people worldwide were living with heart failure at the time. In the United States, heart failure is a major cause of people over 65 being admitted to the hospital. This may be because people are living longer with heart disease due to advancement of treatment of ACS, which can lead to heart failure over time. Shortness of breath is the most common symptom of acute heart failure. From there, the condition shares many of the same symptoms as chronic or severe heart failure. These symptoms may be much more pronounced with acute heart failure though. Swelling of legs and abdomen may occur suddenly and can rapidly gain weight from retaining fluid. It means 2 to 3 pounds in a 24-hour period, or 5 pounds over the course of a week. Patient may also feel nauseous or lose appetite. Other symptoms of both acute and chronic heart failure include weakness, fatigue, irregular or fast heartbeat, coughing and wheezing, decreased ability to concentrate. If not treated, heart failure can lead to a re-infarction. The coronary stenosis prevents oxygen from reaching the myocardium, leading it to pump erratically or not at all. Older adults may have several health conditions. This can make it difficult to isolate symptoms of a heart problem from those caused by other conditions. According to a 2008 study, people hospitalized 
with acute heart failure had an average delay time of 13.3 hours between noticing symptoms and getting treatment. The faster the identification of symptoms and give medical attention, the better the outcome. Color Doppler echocardiography is the key investigation to identify the acute systolic heart failure. For the quantification of systolic heart failure diagnosis, Ejection fraction (EF) can be measured by Simpson method (EF $>30 \%$ was taken as severe systolic heart failure). But EF measurement is very variable as it is operator dependent. Heart failure (HF) is a clinical syndrome that can result from any structural or functional cardiac disorder that impairs the ability of the ventricle to fill with or eject blood. ${ }^{1}$ Transthorasic echocardiography (TTE) is the "gold standard" demonstrates a dilated left ventricle with a reduced ejection fraction and assessment of LV systolic dysfunction and in the recognition of systolic heart failure. Dilatation of the LV with underlying cardiac physiology and clinical status of patients and follow-up of heart disease is the best interpreted by TTE. The functional mitral regurgitation is a consequence of adverse LV remodeling that occurs with a structurally normal valve and it is a marker of adverse prognosis. ${ }^{2,3}$ Severe tricuspid regurgitation (TR) develops in $10-30 \%$ of patients with pulmonary hypertension $(\mathrm{PH})$ and early death in $1 / 3$ such patients within one year. ${ }^{4}$ In systolic heart failure, however, echocardiography has many other roles beyond the recognition of systolic heart failure since dilatation of the LV results in alteration of intracardiac geometry and hemodynamic leading to increased morbidity and mortality. ${ }^{5}$

Pulsus alternans is a physical finding with arterial pulse waveform showing alternating strong and weak beats. It is almost always indicative of left ventricular systolic impairment, and carries a poor prognosis. Pulsus alternans is found in Severe heart failure patients. Pulsus alternans is a poor prognostic sign of severe heart failure patients in acute MI setting. As it is detected by subjective feeling of the physician, so it can be missed in few patients. Another way of record of pulsus alternance is invasive. Arterial puncture is done and catheter is placed to detect the pressure wave in the monitor which is more accurate. But this procedure is expensive and needs expert hand, modern facilities. ${ }^{6}$ Doppler alternans is an objective evidence of Pulsus alternance noninvasively by Echocardiography.

Traditionally, the sensitivity and specificity of a new diagnostic test are derived from its application to two groups of individuals known to have or not to have met the criterion to be detected as judged by means of a "gold standard", some external time-honored test. In this study, a test with unknown accuracy parameters was used to detect severe systolic failure of LV. Since there is a gold standard of detecting systolic failure by EF, so we could compare with Doppler alternance and EF to assess the accuracy of this test, equations were derived relating the sensitivity and specificity of the test to data frequencies based on replicate measurements. The solutions to these equations also provided estimates of the incidence rate of the criterion under investigation in the group tested, and of the predictive values of the test. The validity of this method of estimation will be discussed, and applications to other situations are suggested. ${ }^{7}$

In this study, considering all ethical issues, Color Doppler echocardiography will be done to diagnose and quantify the systolic heart failure. As Doppler alternans was found in severe LV systolic dysfunction, sensitivity and specificity of Doppler alternans was calculated for diagnosis of severe LV systolic failure who needs prompt treatment. So that operator dependent variability could be avoided. This practical demonstration would be the key to enrich our experience and knowledge in the field of acute heart failure in acute coronary syndrome setting.

\section{Materials and Methods}

A. Study place: Division of Heart Failure, Rehabilitation and preventive cardiology, Department of Cardiology, BSMMU, Shahbagh, Dhaka

B. Study population: Patients who admitted in CCU with Acute coronary syndrome with heart failure in department of Cardiology, BSMMU.

C. Study population-

a) Sample Size: 100 (one hundred) patients

b) Age: Between 18 to 70 yrs.

D. Study period: July 2018 to June 2019.

E. Study design: Cross sectional observational study.

F. Selection criteria:

a) Inclusion criteria: Acute coronary syndrome with NYHA class III and IV heart failure

b) Exclusion criteria:

- The patient who was not willing to participate in the study. (Non-consenting patient)

- Frequent PVCs in the form of Bigemini

- Atrial Fibrillation

G. Method of study:

After history taking of 100 Acute MI with heart failure NYHA -III and NYHA -IV Patients, proper examination of 
Cardiovascular system examination was done. Data was collected in Data collection sheet. Pulsus alternans is a physical finding with arterial pulse waveform showing alternating strong and weak beats. It is almost always indicative of left ventricular systolic impairment, and carries a poor prognosis. Color Doppler echocardiography was done in study population to detect cause of Acute heart failure by GE Echocardiography machine. For the quantification of systolic heart failure of LV, Ejection fraction (EF) was measured by Simpson method (EF > $30 \%$ will be taken as severe systolic heart failure) and Doppler study was done in all valves by GE Vivid 7 Ultrasound Machine with a transducer M4S. Single operator has performed the color Doppler echocardiography. Image was taken in B-mode along with spectral Doppler and color flow imaging. Doppler flow was recorded and velocity was measured by $\mathrm{m} / \mathrm{sec} .3$ consecutive Doppler displays was taken for the patient with sinus rhythm. Average of these measurements was taken. There was very little variation of Doppler spectral flow peak in beat to beat. If this variation was more in every alternate beat then it was called "Doppler alternans". Doppler Alternans is also found in frequent premature ventricular ectopics (PVCs) in Bigemini form patients. In this study, Frequent PVCs in Bigemini form patients are excluded. Measurement of EF varies from operator to operator as Simpson method needs skill hand. Doppler study was done to quantify the valvular regurgitation both in MV and TV. Velocity Maximum (Vmax) was measured in Doppler flow which was expressed in meter/sec and Maximum peak gradient(max PG) was also measured in $\mathrm{mm}$ of $\mathrm{Hg}$. Doppler alternans means variation in highest velocity and peak gradient across the valvular regurgitation in every alternate beat. Doppler alternans was identified in severe LV systolic dysfunction with sinus rhythm patients then it could alternative to identify the severe LV systolic dysfunction. In case of severe right heart systolic dysfunction those had TAPSE less than $10 \mathrm{~mm}$, resembles RV EF $<30 \%$ and got significant tricuspid regurgitation also had Doppler alternans in tricuspid valve Doppler study. So, Doppler alternans has also found in severe right heart systolic failure as well as severe left heart systolic failure. Pulsus alternans is found in Severe heart failure patients. Pulsus alternans is a poor prognostic sign of severe heart failure patients in acute MI setting. As it is detected by subjective feeling of the physician, so it can be missed in few patients. Another way of record of pulsus alternans is invasive. Arterial puncture is done and catheter is placed to detect the pressure wave in the monitor which is more accurate. But this procedure is expensive and needs expert hand, modern facilities.

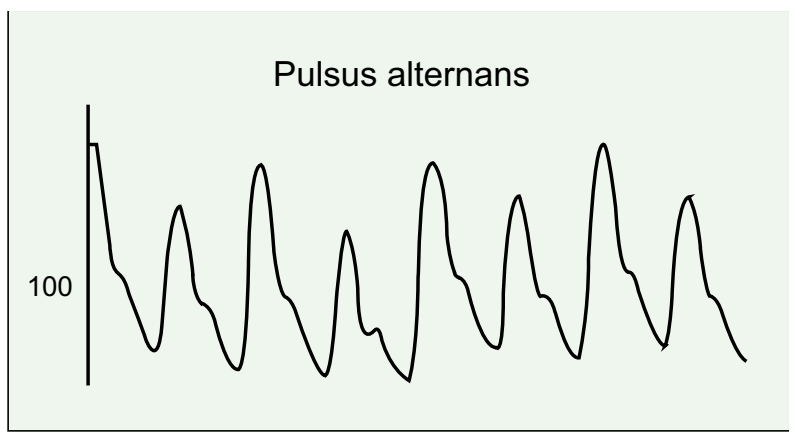

Fig.-1: Pulsus alternans in Pulse wave tracing by Invasive arterial monitoring

This study aimed to detect Doppler alternans in severe left ventricular systolic failure in ACS with heart failure patients. This is an objective evidence of Pulsus alternans noninvasively by Echocardiography. In case of severe right heart systolic failure, significant number of Doppler alternans has been found in tricuspid regurgitation Doppler as incidental finding. So, Doppler alternans has also found in severe right heart systolic failure as well as severe left heart systolic failure. Sensitivity and specificity of Doppler alternans will be measured by following formulas:

Sensitivity $=$ number of true positives number of true positive + number of false negatives

$=$ number of true positives

Total number of sick individual in population

$=$ probability of a positive test given that the patient has the disease

Specificity $=$ number of true negatives number of true negatives + number of false positives

$=$ number of true negatives

Total number of well individual in population

$=$ probability of a negative test given that the patient is well

\section{H. Data collection Procedure}

100 patients were recruited in this study. All data were noted in pre formatted data sheet as approved by Institutional Review board of BSMMU. All relevant Echo report and printouts were kept for analysis.

\section{Statistical Analysis:}

Parametric data were evaluated by independent t- test and categorical data were evaluated by Chi square test. 
Significance were ascribed as $\mathrm{p}$ value $<0.05$. Multivariate regression Analysis has been done by SPSS 16.0 for windows software. Categorical data has been expressed in percentage or number. Parametric datas are expressed in mean +- SD. Sensitivity and specificity of Doppler alternans was calculated in Severe LV systolic heart failure.

\section{Results:}

100 ACS with NYHA class III and IV patients were included in this study. Pie chart 1 showed gender distribution among the study group; Male is 79 and Female is 21. On examination, Pulse was normal in 18 patients, tachycardia 41 patients, bradycardia in 24 and Atrial fibrillation in 17 patients in study group in Table 1. Pulsus alternans was found in 32 patients, 39 had low volume and 29 had normal volume pulse (table 2). More that $50 \%$ patients were hypotensive and 13 patients were hypertensive (table 3 ). Table 4 showed about $50 \%$ patients had ST depression and $\mathrm{T}$ inversion and diagnosed as case of Unstable angina or Non STEMI, 44 had STEMI and 7 had developed new onset of LBBB. Some patients of ACS were presented with arrhythmia, like 17 had PVCs, 5 had short run VT, 8 had VT, 8 had second degree heart block and 13 patients had complete heart block. Figure 2 and 3 showed EF was detected by Modified Simpson method. 87 Patients who have EF $<30 \%$ was marked as Severe LV systolic dysfunction. Among them, (in table 7) 3 had mild, 14 had moderate and rest 70 had severe Mitral regurgitation in color Doppler echocardiography. 38 (43.67\%) patients had Doppler alternans in severe LV systolic dysfunction group. In Figure 4, Apical 4 Chamber view showed alternative change of Doppler in Mitral regurgitation in ' $\mathrm{X}$ ' patient with poor LV systolic dysfunction. So it is the objective evidence of pulsus alternans by echocardiography. Figure 5 also showed similar findings of Doppler alternans across Mitral valve in ' $Y$ ' patient with severe LV systolic dysfunction. In Table 9, $\mathrm{P}$ value for Doppler alternans is 0.039 which is significant. Table 10 showed Doppler alternans by Echocardiography in Severe LV systolic dysfunction patients showed $49 \%$ sensitivity and $78 \%$ specificity. Doppler alternans is not present in all severe LV systolic dysfunction patients. But those who had Doppler alternans must have severe LV systolic dysfunction. These findings are similar in case of RV systolic failure in Table 11. 38 patients were detected as Severe RV systolic dysfunction by $M$ - mode echo measurement of TAPSE $<10 \mathrm{~mm}$. Figure 6 showed the Doppler alternans across Tricuspid valve in case of severe RV systolic dysfunction. Among them 22 (57.89\%) had Doppler alternans in TV Doppler study. Sensitivity of Doppler alternans by Doppler Echocardiography in TV severe RV systolic dysfunction is $51 \%$ and specificity for it is $82 \%$ (Table 12 ).

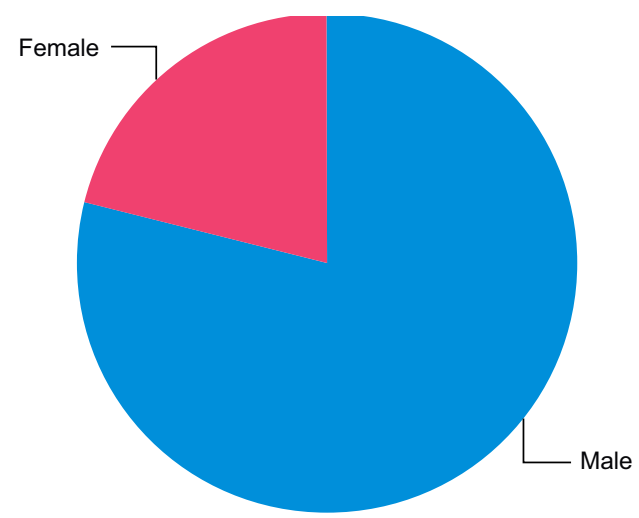

Fig.-2: Gender distribution among study group $(N=100)$

Table-I

Pulse rate on examination

\begin{tabular}{lc}
\hline Pulse rate & Number of patients $(\mathrm{N}=100)$ \\
\hline Normal rate & $18(18 \%)$ \\
Tachycardia & $41(41 \%)$ \\
Bradycardia & $24(24 \%)$ \\
Atrial fibrillation & $17(17 \%)$ \\
\hline
\end{tabular}

Table-II

Pulse volume on examination

\begin{tabular}{lc}
\hline Pulse volume & Number of patients $(\mathrm{N}=100)$ \\
\hline Normal volume & 29 \\
Low volume & 39 \\
Pulsus alternance & 32 \\
\hline \multicolumn{2}{c}{ Table-III } \\
& Blood pressure \\
\hline Blood pressure (BP) & Number of patients $(\mathrm{N}=100)$ \\
\hline Normal & 30 \\
Hypotension & 57 \\
Hypertension & 13 \\
\hline
\end{tabular}

Table-IV

ECG findings

\begin{tabular}{lc}
\hline ECG findings & Number of patients $(\mathrm{N}=100)$ \\
\hline Sinus tachycardia & 41 \\
Sinus bradycardia & 11 \\
UA/ NSTEMI & 47 \\
STEMI & 44 \\
LBBB & 9 \\
RBBB & 11 \\
PACs & 3 \\
PVCs & 17 \\
Short run VT & 5 \\
VT & 8 \\
AF & 17 \\
$1^{0}$ AV block & 4 \\
$2^{0}$ AV block & 8 \\
Complete heart block & 13 \\
\hline
\end{tabular}


Table-V

Findings of AMI by ECG and biomarkers

\begin{tabular}{lc}
\hline Acute STEMI $(\mathrm{n}=88)$ & No of patients \\
\hline Anterior STEMI & 22 \\
Antero septal STEMI & 28 \\
Antero Lateral STEMI & 8 \\
Inferior STEMI & 21 \\
RV infarction & 9 \\
\hline
\end{tabular}

Table-VI

Ejection Fraction (EF) by Simpson method

\begin{tabular}{lc}
\hline EF by Simpson method & No of patient $(\mathrm{N}=100)$ \\
\hline$>30 \%$ & 13 \\
$<30 \%$ & 87 \\
\hline
\end{tabular}

Table-VII

Echo finding of different type Mitral regurgitation in 87 ACS with NYHA class-II and Class -IV patients who had $\mathrm{EF}<30 \%$

\begin{tabular}{lc}
\hline $\mathrm{EF}<30 \%$ (Simpson method) $\mathrm{n}=87$ & No of patient $(\%)$ \\
\hline Mild Mitral regurgitation & $3(3.44 \%)$ \\
Moderate Mitral regurgitation & $14(16.09 \%)$ \\
Severe Mitral regurgitation & $70(80.45 \%)$ \\
\hline
\end{tabular}

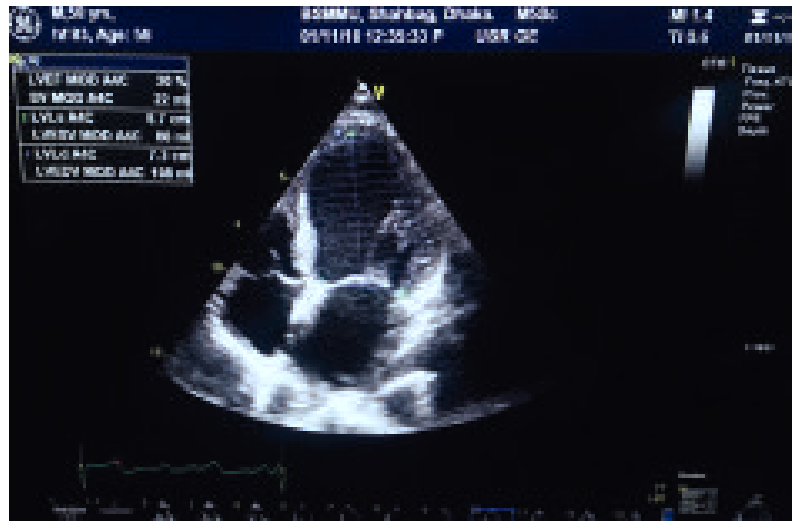

Fig.-3: Severe LV systolic dysfunction by Biplane Simpson method in apical 4 chambers view

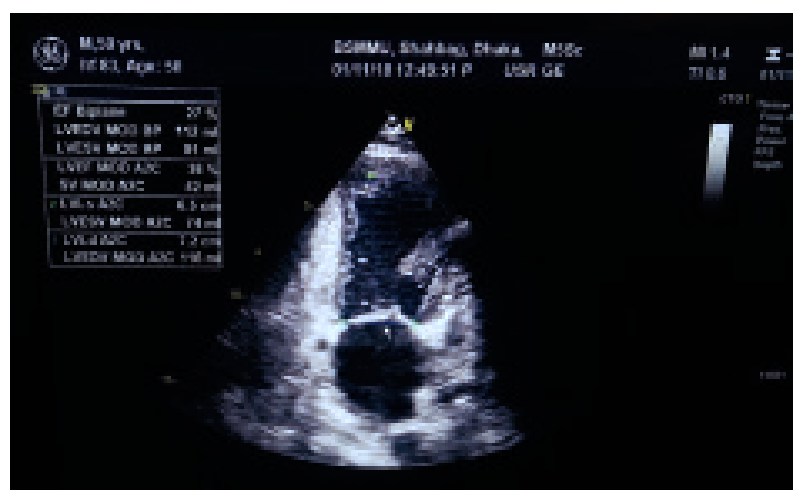

Fig.-4: Biplane Simpson apical 2 chambers view
Table-VIII

Finding of Color Doppler Echocardiography

\begin{tabular}{lcc}
\hline Severe LV systolic dysfunction $(\mathrm{n}=87)$ & No. of Patients $(\%)$ \\
\hline $\begin{array}{l}\text { Similar doppler peak in every beat } \\
\text { Doppler aulternance in (MR peak gradient) }\end{array}$ & $38(56.32 \%)$ \\
\hline \multicolumn{4}{c}{ Table-IX } \\
& Significant P value \\
\hline $\begin{array}{l}\text { Severe LV systolic } \\
\text { dysfunction } \\
\text { (n=87) }\end{array}$ & $\begin{array}{c}\text { Pulsus } \\
\text { alternans } \\
\text { (subjective }\end{array}$ & $\begin{array}{c}\text { Doppler } \\
\text { alternans } \\
\text { (objective }\end{array}$ \\
\hline 87 & $\begin{array}{c}\text { evidence by } \\
\text { pulse) }\end{array}$ & Echocardiography) \\
\hline
\end{tabular}

Table-X

Sensitivity and Specificity of Doppler alternans in Severe LV systolic failure $(E F<35 \%)$

\begin{tabular}{lcc}
\hline Test finding & Sensitivity & Specificity \\
\hline Doppler alternans by & $49 \%$ & $78 \%$ \\
Echocardiography in & & \\
Severe LV systolic & & \\
dysfunction & & \\
\hline
\end{tabular}

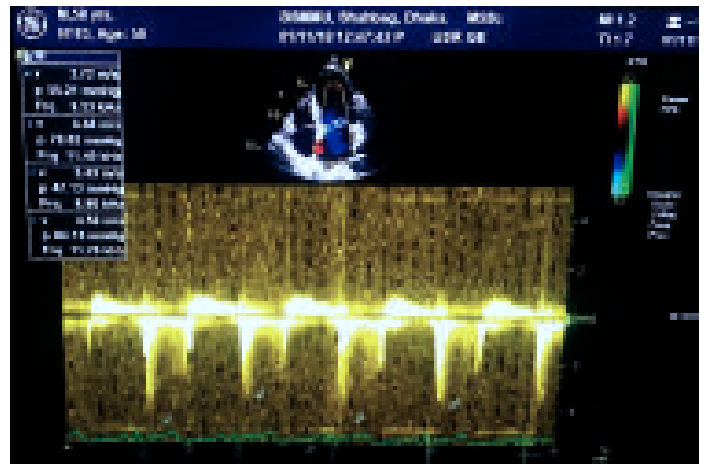

Fig.-5: Apical 4 Chamber view showed alternative change of Doppler in Mitral regurgitation in severe $L V$ dysfunction ' $X$ ' patient

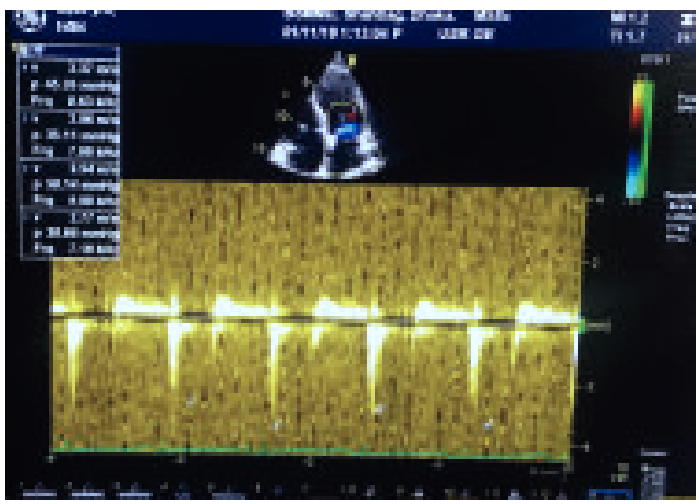

Fig.-6: Apical 4 Chamber view showed alternative change of Doppler in Mitral regurgitation in severe $L V$ dysfunction ' $Y$ ' patient 
Table-XI

Severe RV systolic dysfunction TAPSE $<10 \mathrm{~mm}$

\begin{tabular}{cc}
\hline $\begin{array}{c}\text { Severe RV systolic dysfunction } \\
\text { (TAPSE }<10 \mathrm{~mm})\end{array}$ & $\begin{array}{c}\text { Doppler alternans of Color Doppler Echo } \\
\text { (TR peak gradient) }\end{array}$ \\
\hline 38 & $22(57.89 \%)$ \\
\hline
\end{tabular}

Table-XII

Sensitivity and Specificity of Doppler alternans in Severe RV systolic dysfunction (TAPSE $<10 \mathrm{~mm}$ )

\begin{tabular}{lcc}
\hline Test finding & Sensitivity & Specificity \\
\hline Doppler alternans by Doppler Echocardiography in TV severe RV systolic dysfunction & $51 \%$ & $82 \%$ \\
\hline
\end{tabular}
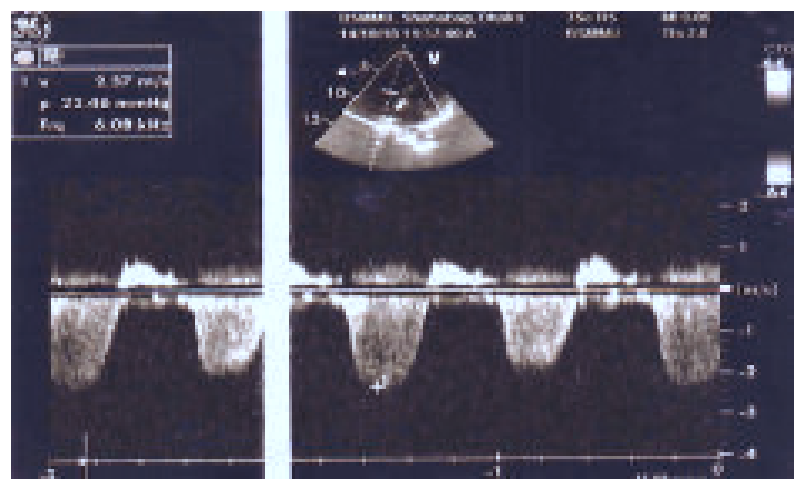

Fig.-7: Apical 4 Chamber view showed alternative change of Doppler in Tricuspid regurgitation

\section{Discussions:}

Pulsus alternans is usually found in severe left ventricular systolic dysfunctions due to coronary artery disease, ${ }^{7}$ systemic hypertension, ${ }^{7}$ cardiomyopathy ${ }^{9}$ and aortic stenosis. ${ }^{10}$ It has also been reported in acute transient ischemia and in patient with normal heart during or after supraventricular tachycardia ${ }^{11}$ In this study, 32 patients with ACS with heart failure had pulsus alternans on examination of pulse. There has been continuing interest in understanding the mechanisms and clinical manifestations of pulsus alternans since its first description by Traube in 1872, although its cause and exact mechanism are poorly understood. Two main mechanisms have been proposed to account for pulsus alternans. The first, based on the Frank Starling mechanism, proposes beat-to-beat alteration in end-diastolic volume accounted for the change in force of contraction. ${ }^{14,16}$ Impaired systolic contraction of a failing ventricle reduces stroke volume, resulting in an elevated end diastolic volume for the next contraction. Elevated end diastolic volume results in increased myofibril length and therefore, increased contraction on the next beat. ${ }^{14,16} \mathrm{An}$ increase heart rate accentuates this process as diastolic filling is further impaired. Although this mechanism may be contributory, the popularity of this mechanism has waned over the recent years as there is experimental evidence which suggests the absence of alternation in end diastolic volume with the stronger beats of pulsus alternans. ${ }^{17}$ Also in animal studies, pulsus alternans has been produced and maintained with constant preload and lack of alternation in mitral inflow. ${ }^{11}$ The alternation in the contractile state of the myocardium, the "myocardial theory," underlies some instances of pulsus alternans but in no way negates the possibility that FrankStarling principle accounting for some instances of pulsus alternans. ${ }^{17}$ The second proposed mechanism which explains the cause of pulsus alternans is the alteration in cellular handling of calcium during the cardiac contractility. ${ }^{18,19}$ The physiological action involves the abnormality of intracellular calcium cycling, involving the sarcoplasmic reticulum. ${ }^{18}$ Calsequestrin is a protein in the inner membrane surface of the sarcoplasmic reticulum that binds and stores calcium. Schmidt et al ${ }^{18}$ found that mice that overexpressed calsequestrin had pulsus alternans during high heart rates. Another study showed that echocardiographic evidence of Pulsus alternans is commonly found in severe left ventricular systolic dysfunctions. ${ }^{20}$ The study concluded that the mice had a delay between the uptake and release of calcium from the sarcoplasmic reticulum and this alteration in calcium cycling and use led to development of pulsus alternans in vivo. Here in this study, about 87 (n) patients had severe LV systolic dysfunction by calculating Ejection fraction (EF) in Simpson method. Among them 38 (43.67\%) had Doppler alternans in Mitral valve Doppler study. But on examination, only 32 patients had pulsus alternans by subjective feeling of pulse. As pulsus alternans is a bad prognostic marker of heart failure patients. It is important to find out number of those patients who have pulsus alternans. So that appropriate measure can be taken and early treatment can be given in time to prevent 
cardiovascular mortality and morbidity and frequent hospitalization. As Doppler alternans is the objective evidence of pulsus alternans and it can be found noninvasively by Echo Doppler study. Right ventricular alternans is usually associated pulsus alternans on the left side. ${ }^{9}$ Isolated right ventricular alternans seems to be related to right ventricular dysfunctions and increased pulmonary resistance due to reactive air way disease, ${ }^{7}$ pulmonary embolism ${ }^{12}$ and pulmonary hypertension. ${ }^{13}$ In present study, 38 patients had right ventricular severe systolic dysfunction which was identified by TAPSE in M mode echocardiography. If TAPSE is found $<10 \mathrm{~mm}$ then it is considered as severe RV systolic dysfunction. Among them, 22(57.89\%) patients had Doppler alternans in TV color Doppler study. Massive pulmonary embolisms can cause acute cor pulmonale and can lead to right ventricular alternans; however, it has not known to cause biventricular alternans. Biventricular alternans is rare, and only few cases have been described in patient with severe left ventricular dysfunction and left anterior descending coronary artery disease. ${ }^{9,14}$ Cournand et al reported biventricular alternans in 9 patients, most of whom had dilated cardiomyopathy, systemic hypertension, congestive heart failure and myocardial fibrosis. ${ }^{15}$ It has observed that only 5 patients had biventricular alternance in this study group patient.

\section{Conclusions:}

Isolated left and right ventricular alternans usually occur in severe heart failure. Biventricular Doppler alternans is a rare phenomenon and has only been described in few cases of severe left ventricle systolic dysfunction. In most cases, pulsus alternans is an ominous sign that suggests severe heart failure and poor prognostic sign of this group of patients. Doppler alternans is an objective evidence of it by noninvasive Echocardiography Doppler study. Early recognition can aid in appropriate management and intervention, which may change patient outcome.

\section{References:}

1. Braunwald E. Heart disease. A textbook of cardiovascular medicine. Sanduers; 9th edition 2007; p-487.

2. Lamas GA, Mitchell GF, Flaker GC, Smith SC, Jr, Gersh BJ, Basta L, Moye L, Braunwald E, Pfeffer MA. Clinical significance of mitral regurgitation after acute myocardial infarction. Circulation. 1997;96:827-33.

3. Lancellotti P, Gerard PL, Pierard LA. Long-term outcome of patients with heart failure and dynamic functional mitral regurgitation. Eur Heart J. 2005;26:1528-1532. doi: 10.1093/ eurheartj/ehi189.

4. Koelling TM, Aaronson KD, Cody RJ, Bach DS, Armstrong WF. Prognostic significance of mitral regurgitation and tricuspid regurgitation in patients with left ventricular systolic dysfunction. Am Heart J. 2002; 144:524-29.

5. St John Sutton M, Pfeffer MA, Plappert T, Rouleau JL, Moyé LA, Dagenais GR, Lamas GA, Klein M, Sussex B, Goldman S, et al. Quantitative two-dimensional echocardiographic measurements are major predictors of adverse cardiovascular events after acute myocardial infarction. The protective effects of Captopril. Circulation. 1994; 89:68-75.

6. Michaels AD, Browne AE, Varghese P, Chou TM. Intracoronary measurement of pulsus alternans. Catheter Cardiovasc Interv 2000; 51: 335-38.

7. Michael Schulzer,Douglas R. Anderson, Stephen M. Drance et al. Sensitivity and specificity of a diagnostic test determined by repeated observations in the absence of an external standard. JCE.1991;44(11): 1167-79.

8. Youden, W.J. "Index for rating diagnostic tests". Cancer. 1950; 3: 32-35.

9. Vidwan P, Stouffer GA. Biventricular pulsus alternans. Cardiol Res Pract 2009; 2009: 703-93.

10. Gagnon RM, Doyle D. Transient left ventricular pulsus alternans in severe aortic valve disease. Can J Cardiol 1988; 4: $217-18$.

11. Edwards P, Cohen GI. Both diastolic and systolic function alternate in pulsus alternans: a case report and review. J Am Soc Echocardiogr 2003; 16: 695-97.

12. Calick A, Berger S. Pulmonary artery pulsus alternans associated with pulmonary embolism. Chest 1973; 64: 663-64.

13. Askew JW, Lee RW. Isolated pulmonary arterial pulsus alternans secondary to pulmonary hypertension. Clin Cardiol 2004; $27: 358$.

14. Surawicz B, Fisch C. Cardiac alternans: diverse mechanisms and clinical manifestations. J Am Coll Cardiol 1992; 20: 483-99.

15. Cournand A, Ferrer MI, Harvey RM, Richards DW. Cardiocirculatory studies in pulsus alternans of the systemic and pulmonary circulations. Circulation 1956; 14: 163-74

16. Lab MJ, Seed WA. Pulsus alternans. Cardiovasc Res 1993; 27 : 1407-12.

17. Cohn KE, Sandler H, Hancock EW. Mechanisms of pulsus alternans. Circulation 1967; 36: 372-80.

18. Schmidt AG, Kadambi VJ, Ball N, Sato Y, Walsh RA, Kranias EG, Hoit BD. Cardiac-specific overexpression of calsequestrin results in left ventricular hypertrophy, depressed forcefrequency relation and pulsus alternans in vivo. $\mathrm{J}$ Mol Cell Cardiol 2000; 32: 1735-44.

19. Kotsanas G, Holroyd SM, Young R, Gibbs CL. Mechanisms contributing to pulsus alternans in pressure-overload cardiac hypertrophy. Am J Physiol 1996; 271: H2490-H2500.

20. Perk G, Tunick PA, Kronzon I. Systolic and diastolic pulsus alternans in severe heart failure. J Am Soc Echocardiogr 2007; 20: 905. e5-e7. 